\title{
SOME CRITERIA OF UNIVALENCE ${ }^{1}$
}

\section{ZEEV NEHARI}

In an earlier paper [3] it was shown that the univalence of an analytic function $w=f(z)$ in the unit disk can be assured by conditions of the type $|\{w, z\}| \leqq m(|z|)$, where $m(|z|)$ is a suitable positive function and

$$
\{w, z\}=\left(\frac{w^{\prime \prime}}{w^{\prime}}\right)^{\prime}-\frac{1}{2}\left(\frac{w^{\prime \prime}}{w^{\prime}}\right)^{2}
$$

is the Schwarzian derivative of $w=f(z)$. The two cases treated in [3] were $m(|z|) \equiv \pi^{2} / 2$ and $m(|z|)=2\left(1-|z|^{2}\right)^{-2}$. The constants appearing in both criteria are the largest possible. In the first case this is shown by the existence of the nonunivalent function $w=\tan \pi(1+\epsilon) z / 2$ $(\epsilon>0)$ for which $\{w, z\}=\pi^{2}(1+\epsilon)^{2} / 2$, and in the second case by an example constructed by E. Hille [2]. Other criteria of this type have meanwhile been announced (without proof) by V. Pokornyi [4], the only sharp one among them being the one corresponding to $m(|z|)$ $=4\left(1-|z|^{2}\right)^{-1}$, with the extremal $f(z)=\int_{0}^{z}\left(1-z^{2}\right)^{-2} d z$.

The main objective of the present note is to establish the following more general criterion of univalence.

Theorem I. The function $f(z)$ will be univalent in $|z|<1$ if

$$
|\{f(z), z\}| \leqq 2 p(|z|) \text {, }
$$

where $p(x)$ is a function with the following properties: (a) $p(x)$ is positive and continuous for $-1<x<1$; (b) $p(-x)=p(x)$; (c) $\left(1-x^{2}\right)^{2} p(x)$ is nonincreasing if $x$ varies from 0 to 1 ; (d) the differential equation

$$
y^{\prime \prime}(x)+p(x) y(x)=0
$$

has a solution which does not vanish for $-1<x<1$. The constant 2 in (1) cannot be replaced by a larger number.

The proof of Theorem I, like that of the other criteria mentioned above, rests on the fact that a function $f(z)$ is univalent in a region $D$ if, and only if, no solution of the differential equation

$$
u^{\prime \prime}(z)+q(z) u(z)=0, \quad 2 q(z)=\{f(z), z\}
$$

vanishes in $D$ more than once [3]. If $f(z)$ is not univalent in $|z|<1$,

Received by the editors December 16, 1953.

${ }_{1}$ This research was sponsored by the United States Air Force, through the Air Research and Development Command. 
there will therefore be two points, say $\alpha$ and $\beta(|\alpha|<1,|\beta|<1, \alpha \neq \beta)$, at which one of the solutions of (3) will vanish. There exists a unique circle which passes through $\alpha$ and $\beta$ and is orthogonal to $|z|=1$. This circle is divided by $|z|=1$ into two arcs, one of which contains the points $\alpha, \beta$ and will be denoted by $C$. Since the statement of Theorem $I$ is invariant with respect to a rotation of the $z$-plane about the origin we may assume, without losing generality, that $C$ is in the upper half-plane and symmetric with respect to the imaginary axis.

A suitable linear substitution of the form ${ }^{2}$

$$
z=\frac{w+\zeta}{1+\zeta^{*} w}
$$

will carry $C$ into the linear segment $-1<w<1$, and it will, of course, map $|z|<1$ onto $|w|<1$. It is easy to see that, because of the particular location of $C$, one of these substitutions must be of the form

$$
z=\frac{w+i \rho}{1-i \rho w}, \quad 0 \leqq \rho<1 .
$$

The points $\alpha, \beta$ are carried, respectively, into two points $a, b$ on the real axis. We may assume, without loss of generality, that $a$ is at the left of $b$, so that $-1<a<b<1$.

The substitution (4) will transform the equation (3) into

$$
v^{\prime \prime}(w)+q_{1}(w) v(w)=0,
$$$$
u(z)=\phi(w) v(w),
$$

where $\phi(w)$ is regular and different from zero in $|w|<1$, and

$$
2 q_{1}(w)=\{g(w), w\}, \quad g(w)=f\left(\frac{w+\zeta}{1+\zeta^{*} w}\right) .
$$

It is easily confirmed that

$$
\{g(w), w\}=\left(\frac{d z}{d w}\right)^{2}\{f(z), z\}
$$

and that

$$
\left|\frac{d z}{d w}\right|=\frac{1-|z|^{2}}{1-|w|^{2}} .
$$

It follows therefore that

$$
\left(1-|w|^{2}\right)^{2}|\{g(w), w\}|=\left(1-|z|^{2}\right)^{2}|\{f(z), z\}|,
$$

2 Asterisks denote complex conjugates. 
and thus, by (1), that

$$
\left(1-|w|^{2}\right)^{2}|\{g(w), w\}| \leqq 2\left(1-|z|^{2}\right)^{2} p(|z|) .
$$

By hypothesis (c) of Theorem I, $\left(1-x^{2}\right)^{2} p(x)$ is nonincreasing if $x$ grows from 0 to 1 . Now it is evident from (5) that $|z|>|w|$ if $-1<w<1$. Hence,

$$
\left(1-|z|^{2}\right)^{2} p(|z|) \leqq\left(1-w^{2}\right)^{2} p(w), \quad-1<w<1,
$$

and therefore

$$
|\{g(w), w\}| \leqq 2 p(w), \quad-1<w<1 .
$$

By our assumptions, there exists a solution $v(w)$ of (6) which vanishes at two points $a, b$ for which $-1<a<b<1$. Multiplying (6) by $v^{*}(w)$ and integrating from $a$ to $b$ along the real axis, we obtain, after an integration by parts,

$$
\int_{a}^{b}\left|v^{\prime}(w)\right|^{2} d w=\int_{a}^{b} q_{1}(w)|v(w)|^{2} d w
$$

Hence, by (7) and (8),

$$
\int_{a}^{b}\left|v^{\prime}(w)\right|^{2} d w \leqq \int_{a}^{b} p(w)|v(w)|^{2} d w .
$$

If we write $v(w)=\sigma(w)+i \tau(w)$, both $\sigma(w)$ and $\tau(w)$ vanish for $w=a, b$, and we have $\left|v^{\prime}(w)\right|^{2}=\sigma^{\prime 2}(w)+\tau^{\prime 2}(w)$. Thus,

$$
\int_{a}^{b}\left[\sigma^{\prime 2}(w)+\tau^{\prime 2}(w)\right] d w \leqq \int_{a}^{b} p(w)\left[\sigma^{2}(w)+\tau^{2}(w)\right] d w .
$$

Let now $\lambda$ be the lowest eigenvalue of the differential system

$$
y^{\prime \prime}(w)+\lambda p(w) y(w)=0, \quad y(a)=y(b)=0 .
$$

By Rayleigh's inequality, we have

$$
\lambda \int_{a}^{b} p(w) \sigma^{2}(w) d w \leqq \int_{a}^{b} \sigma^{\prime 2}(w) d w
$$

and a similar inequality for $\tau(w)$. Combining this with (9), we obtain

$$
\int_{a}^{b}\left[\sigma^{\prime 2}(w)+\tau^{\prime 2}(w)\right] d w \leqq \frac{1}{\lambda} \int_{a}^{b}\left[\sigma^{\prime 2}(w)+\tau^{\prime 2}(w)\right] d w .
$$

It follows that $\lambda \leqq 1$ and therefore, in view of $p(w)>0$ and the Sturm comparison theorern, that a solution of (2) which vanishes at 
$w=a$ must have yet another zero in the interval $a<w \leqq b$. By the Sturm separation theorem, all solutions of (2) must therefore vanish in the interval $a \leqq w \leqq b$, and thus in $-1<w<1$. But this disagrees with hypothesis (d) of Theorem I, and the assumption that there exists a solution of (3) which vanishes in $|z|<1$ more than once will therefore lead to a contradiction. It follows that the function $f(z)$ must be univalent if it satisfies the hypotheses of Theorem I.

If $p(x) \equiv \pi^{2} / 4$, or $p(x)=\left(1-x^{2}\right)^{-2}$, equation (2) has the solutions $y=\cos \pi x / 2$ and $y=\left(1-x^{2}\right)^{1 / 2}$, respectively, and Theorem I applies, yielding the results derived in [3]. Since these results are sharp, the same is thus true of Theorem I. Theorem I is, however, not only sharp in the sense that the constant 2 in (1) cannot in general be replaced by a larger one. The following, more precise, statement holds.

Let $p(z)$ be regular in $|z|<1,|p(z)| \leqq p(|z|)$, and let $p(x)(z=x+i y)$ satisfy hypotheses (a), (b), (c), (d) of Theorem I. If (2) has a solution which vanishes for $x= \pm 1$, and if $\epsilon>0$, then there exists a function $f(z)$ which is not univalent in $|z|<1$ and for which

$$
|\{f(z), z\}|=(2+\epsilon) p(|z|)
$$

for suitable values of $z$.

Indeed, if we set $2 q(z)=(2+\epsilon) p(z)$, the equation $u^{\prime \prime}(z)+q(z) u(z)=0$ will-by the Sturm comparison theorem-have a solution with two zeros in the interval $-1<z<1$. It follows that the function $f(z)$, for which $\{f(z), z\}=2 q(z)$, cannot be univalent in $|z|<1$. Since, moreover, $f(z)$ satisfies (10) for real values of $z$, our statement is proved.

Every function $p(x)$ which satisfies hypotheses (a), (b), (c), and for which we can find the lowest eigenvalue $\lambda$ of the differential system

$$
y^{\prime \prime}(x)+\lambda p(x) y(x)=0, \quad y( \pm 1)=0,
$$

will therefore yield a sharp criterion of univalence, provided $p(z)$ is regular in $|z|<1$ and $|p(z)| \leqq p(|z|)$. For instance, if $p(x)=\left(1-x^{2}\right)^{-1}$, (11) has the solution $y(x)=1-x^{2}$, with $\lambda=2$. $f(z)$ will thus be univalent in $|z|<1$ if $|\{f(z), z\}| \leqq 4\left(1-|z|^{2}\right)^{-1}$, in accordance with the result of Pokornyi [4] mentioned further above.

If (11) cannot be solved explicitly, less accurate criteria can be obtained by estimating the eigenvalue $\lambda$ from below. As an illustration, we replace (11) by the equivalent integral equation

$$
y(\xi)=\lambda \int_{-1}^{1} p(x) g(x, \xi) y(x) d x,
$$


where $2 g(x, \xi)=(1+x)(1-\xi)$ for $-1 \leqq x \leqq \xi$ and $2 g(x, \xi)=(1+\xi)(1-x)$ for $\xi \leqq x \leqq 1$. Obviously, $2 g(x, \xi) \leqq 1-x^{2}$ for $-1 \leqq x \leqq 1$. If $\xi$ is taken to be such that $|y(x)| \leqq|y(\xi)|$ in $-1 \leqq x \leqq 1$, it follows from (12) that

$$
1 \leqq \lambda \int_{-1}^{1} p(x) g(x, \xi) d x,
$$

and therefore

$$
2 \leqq \lambda \int_{-1}^{1} p(x)\left(1-x^{2}\right) d x .
$$

Combining this with Theorem I, we arrive at the following result. If

$$
|\{f(z), z\}| \leqq \frac{2 p(|z|)}{\int_{0}^{1}\left(1-x^{2}\right) p(x) d x}, \quad|z|<1,
$$

and $p(x)$ satisfies hypotheses (a), (b), (c) of Theorem I, then $f(z)$ is univalent in $|z|<1$.

While it is known that the constant 2 in (13) is the largest possible [1], the decision whether or not (14) is the best criterion of its kind will depend on the existence-or non-existence-of functions $p(x)$ for which the right-hand side of (13) is arbitrarily close to 2 and which, at the same time, satisfy $|p(z)| \leqq p(|z|)(|z|<1)$ and hypotheses (a), (b), (c).

\section{REFERENCES}

1. P. Hartman and A. Wintner, On an oscillation criterion of Liapounoff, Amer. J. Math. vol. 73 (1951) pp. 885-890.

2. E. Hille, Remarks on a paper by Zeev Nehari, Bull. Amer. Math. Soc. vol. 55 (1949) pp. 552-553.

3. Z. Nehari, The Schwarzian derivative and schlicht functions, Bull. Amer. Math. Soc. vol. 55 (1949) pp. 545-551.

4. V. V. Pokornyi, On some sufficient conditions for univalence, Doklady Akademii Nauk SSSR (N.S.) vol. 79 (1951) pp. 743-746.

WASHINGTON UNIVERSITY 